This item was submitted to Loughborough's Research Repository by the author.

Items in Figshare are protected by copyright, with all rights reserved, unless otherwise indicated.

\title{
The development of a visualisation tool for acquired motorsport data
}

PLEASE CITE THE PUBLISHED VERSION

http://dx.doi.org/10.1177/1754337115615256

\section{PUBLISHER}

Sage / @ IMechE

\section{VERSION}

AM (Accepted Manuscript)

\section{PUBLISHER STATEMENT}

This work is made available according to the conditions of the Creative Commons Attribution-NonCommercialNoDerivatives 4.0 International (CC BY-NC-ND 4.0) licence. Full details of this licence are available at: https://creativecommons.org/licenses/by-nc-nd/4.0/

\section{LICENCE}

CC BY-NC-ND 4.0

\section{REPOSITORY RECORD}

Parker, Matthew C., and Graham K. Hargrave. 2019. "The Development of a Visualisation Tool for Acquired Motorsport Data". figshare. https://hdl.handle.net/2134/20073. 


\section{Original Article}

\section{Corresponding author:}

Wolfson School of Mechanical and Manufacturing Engineering, Loughborough University, Loughborough, UK

Email: m.parker@lboro.ac.uk

\section{Title}

The development of a visualisation tool for acquired motorsport data

\section{Authors}

Matthew C Parker and Graham K Hargrave

Wolfson School of Mechanical and Manufacturing Engineering, Loughborough University, Loughborough, UK

\section{Abstract}

Data acquisition and analysis are an intrinsic part of motorsport, helping a race team objectively evaluate the performance of both their car and driver. Over time, data acquisition has become almost universally employed through all levels of racing. Whilst large teams in the sport's highest ranks have many resources to derive answers from this data, users in more minor 'stepping stone' categories often find themselves unable to successfully exploit the full potential of the information gathered because of its volume and their limited resources. 
Further to issues associated with the volume of data, recent trends in racing have seen cuts to the time available for driver and car testing through all levels of the sport to reduce escalating competition costs.

Whilst users are faced with ever more data and less time in which to extract useful information, the tools provided by commercial analysis packages have shown little development. This paper describes the investigation into a new three dimensional graphical display method, which aims to help the user more rapidly assimilate acquired motorsport data to the race car producing it. The first two preliminary stages of development of this system are presented, demonstrating the ability of the system to operate with two levels of complexity, which might be considered to suitably represent different levels of user. Together, results from both demonstrate the system's potential for further development as a useful tool for accelerating a race team's analysis of acquired data.

\section{Key words}

Data acquisition, data analysis, motorsport, assimilation, graphical display, optical sensor

\section{Background}

Motor racing is a highly competitive sport contested at a range of levels. Below the wellpublicised upper echelons of the sport, such as Formula 1 and sports prototype racing, a vast number of teams and drivers compete in club level events and also in other smaller professional series feeding into the sport's higher categories. Universal amongst all but a small fraction of these competitors is the use in some form of data acquisition and analysis [1, 2]. 
A typical motorsport data acquisition system features a data logging unit, recording vehicle speed, acceleration, driver control inputs, suspension movement, and various engine performance measures, such as temperatures and pressures. The recorded data will be used by a team to analyse performance of both the vehicle and driver. In response to the findings of this analysis, there are various performance adjustments which can be made to a racing car and various aspects of a driver's approach which can be modified to achieve a faster lap time. A reduction in hardware costs have made data acquisition and analysis available to an increasingly broad range of users, including those teams in the sport's club, national and some international feeder series. The numbers of personnel available in these categories, dictated by available funding, makes the task of fully analysing and understanding acquired data difficult. As a result, many users of motorsport data acquisition are unable to fully utilise the data they collect $[3,4]$.

Further to this previous point, in recent years there have been increasingly strict limits imposed on the amount of time teams can test $[5,6]$. This has exacerbated any shortcomings of a team, as the importance of all available testing time has increased. A net result has been that often data has not been used effectively or at all during a test session as running of the car on track has been prioritised over reasoned and deliberate data analysis; the potential recorded data offers for steering the development of the driver and car has therefore been lost [4].

Also of importance is the fact that recorded data itself provides a focal point for communication between a team or coach and the driver and should therefore ideally be presented in a way that accounts for the differing technical levels of each. It could be expected that the development of 
data analysis software should have matched that of the data acquisition hardware, however a review of commercially available software making up part of the work summarised here indicated that the level of current software left considerable scope for development [7]. The tools highlighted across this range were mainly tabular or graph-based and have essentially been in place throughout the existence of data acquisition and analysis in motorsport. A graphbased display provides valuable information, particularly with regards to analysing large scale trends in vehicle/driver performance. However, there are limitations to this approach, particularly when considering close scale events and the phenomena causing them, which can often involve consideration of multiple data channels.

A number of small camera systems are currently available to image a vehicle's movement whilst on the race track providing some visualisation which can be compared to recorded data plotted on graphs to assist the user's assimilation of the information. Pi Research, for example, markets a typical system entitled VIDS [8]. This system incorporates an in-car camera system to enable the vehicle positioning to be reviewed during the lap. This system can be combined with Pi Research's data analysis packages to enable a visual display to be synchronised with vehicle data. However, this system is limited by the camera frame rate and the single frame of reference for the imaging data.

Moloney et al. [9] reported the use of high speed cameras at the track side, used to gather visual information for reference, measurement and assimilation purposes. The high speed cameras' frame rates allowed for the capture of dynamic events in the car's suspension and chassis behaviour which were beneficial to understanding the overall vehicle performance. However, there were issues involved in this approach, relating to the cost of camera equipment 
and the practicality of covering the whole circuit and of processing the collected video. It is therefore clear that whilst video of a race car can offer additional insight and aid assimilation of data, there are possible practicality issues associated with its use. A network of cameras to enable multiple fixed frame of reference views becomes expensive and complex to deploy. Lighting as well as camera lensing effects, such as distortion, perspective and focus could all also potentially impact the results.

An alternative to video capture was found to exist in the area of aircraft pilot training. A number of software systems exist to recreate a flight as a three dimensional animation, based on Global Positioning System (GPS) data and recorded measurements of the aircraft's attitude $[10,11$, 12]. An alternative to filming the car on circuit would therefore be to graphically recreate its track outing from the data recorded by its data acquisition system, a similar vein. This approach would enable an infinite number of viewpoints to be recreated, based directly on captured data rather than from large video files.

The concept of applying an approach of a visually realistic three dimensional (3D) data recreation to motorsport data was conceived as a useful step forward for assisting the analysis of acquired vehicle data at the race track. The remainder of this paper describes the implementation of a 3D animation of data recorded from a racing car and the complexities associated with positioning the vehicle and recreating its 3D attitude. The approach described involves combining both existing and new methods into a visualisation system, which has yet to be published, for more effective racing car data display. This was developed to primarily accept universally collected data, making it applicable to all levels of motorsport, but also to optionally utilise additional sensor measurements, likely to be collected by higher level teams. 


\section{Development of the visualisation tool}

The development of a visualisation tool for motorsport applications was completed in two main stages. Firstly a graphics routine was developed to present motorsport data in a format allowing the user to assimilate recorded data to the vehicle more easily and hence additionally aid communication between data analyst and driver. To demonstrate the benefits of this approach within motorsport, wheel speed and lateral acceleration data captured from a World Series by Renault race car was used. The second stage focussed on developing code to analyse and display suspension displacements, steering angles, ride height, vehicle attitude and ground displacement data recorded using a new sensor technology.

The remainder of this section introduces the modelling and animation of the visualisation tool, which was essentially common to the two versions of the application. The subsequent sections then describe the two different versions of the visualisation tool, focussing on the data analysis routines for the purpose of vehicle animation and the results that can be obtained from this novel visualisation tool.

\subsection{Graphical modelling of test vehicles}

The data used for testing the first version of the application was provided through a link with P1 Motorsport and came from a World Series by Renault (WSbR) FR3.5 car navigating the Donington Park GP circuit. In order to create a realistic vehicle model, information available from the WSbR technical support service was used to create suspension component and 
chassis models in the Solid Edge ${ }^{\circledR}$ Computer Aided Design (CAD) package $[13,14]$. This included broad chassis dimensions as well as 3D coordinates of suspension joint locations; information that is readily available to all teams competing in the series. The vehicle model was then converted to a polygon format 3ds model using 3D Studio Max® for drawing with OpenGL® (Open Graphics Library); an approach which enabled fast drawing and face culling to ensure fast execution of the final display [14].

The second development stage used a kitcar, manufactured by Robin Hood Sports Cars, as a test vehicle to allow for testing of sensor technology developed alongside. This vehicle's suspension was measured with a FaroArm ${ }^{\circledR}$ Coordinate Measurement Machine (CMM), whilst the bodywork was measured with PhotoModeler $5 \circledR$, a Photogrammetry based software application for 3D modelling, in the absence of any available measurement data. Both vehicles are illustrated in the results sections presented later.

\subsubsection{Animation}

The visualisation application was designed for a time restricted working environment.

Therefore, development was undertaken in Visual C++ and using OpenGL $®$ graphics libraries to ensure fast execution times $[15,16,17]$. OpenGL $®$ operates a simple polygonal drawing regime, creating graphics by drawing flat polygons between defined points in space. Polygonal model formats contain a list of vertex data which defines a collection of polygons that recreate the surface geometry of the model. The 3ds file format was used here with a model loader class to read each of the model's polygons in turn before calling the primitive OpenGL® functions to draw those to screen. 
The animation playback was controlled by a Visual C++ application and allowed the user full flexibility to view the recorded data at any playback speed and from any angle without the need for either a network of cameras or lengthy simulation. It also presents the opportunity to visualise dynamic aspects of the vehicle's behaviour which cannot be accessed by cameras. Graphed plots of data were also featured alongside the animation, since this new display approach was intended to be complementary to existing methods.

\section{Preliminary visualisation application}

The first tests of the system were based upon data collected from a real racing car, as described previously. Although from a high level of motor racing, this data represented the most basic data streams that could be used with the visualisation tool. This section describes firstly how the vehicle's trajectory was estimated from this data and then describes the results of the system testing.

\subsection{Trajectory reconstruction/vehicle model positioning}

An initial starting point for positioning the vehicle model in a 3D environment, in order to create the visual impression of it travelling, was to approximate its trajectory across a flat plane and use this as the basis of positioning the graphical model during animation. It is standard for racing car data acquisition systems to measure the vehicle's forward speed derived from a Hall Effect type sensor facing a moving trigger plate attached to a front wheel as well as lateral acceleration, measured by an accelerometer mounted close to the vehicle's centre of gravity [18]. These two streams of data are commonly combined to derive an approximate vehicle 
trajectory using a method described by [19] across all data analysis packages as an option alongside GPS data, or indeed the sole method for approximating a plan view reconstruction of the car's trajectory around the circuit $[2,20]$. The method is fully described by [19] but is briefly presented here for completeness alongside a diagram illustrating the terms used (Figure 1).

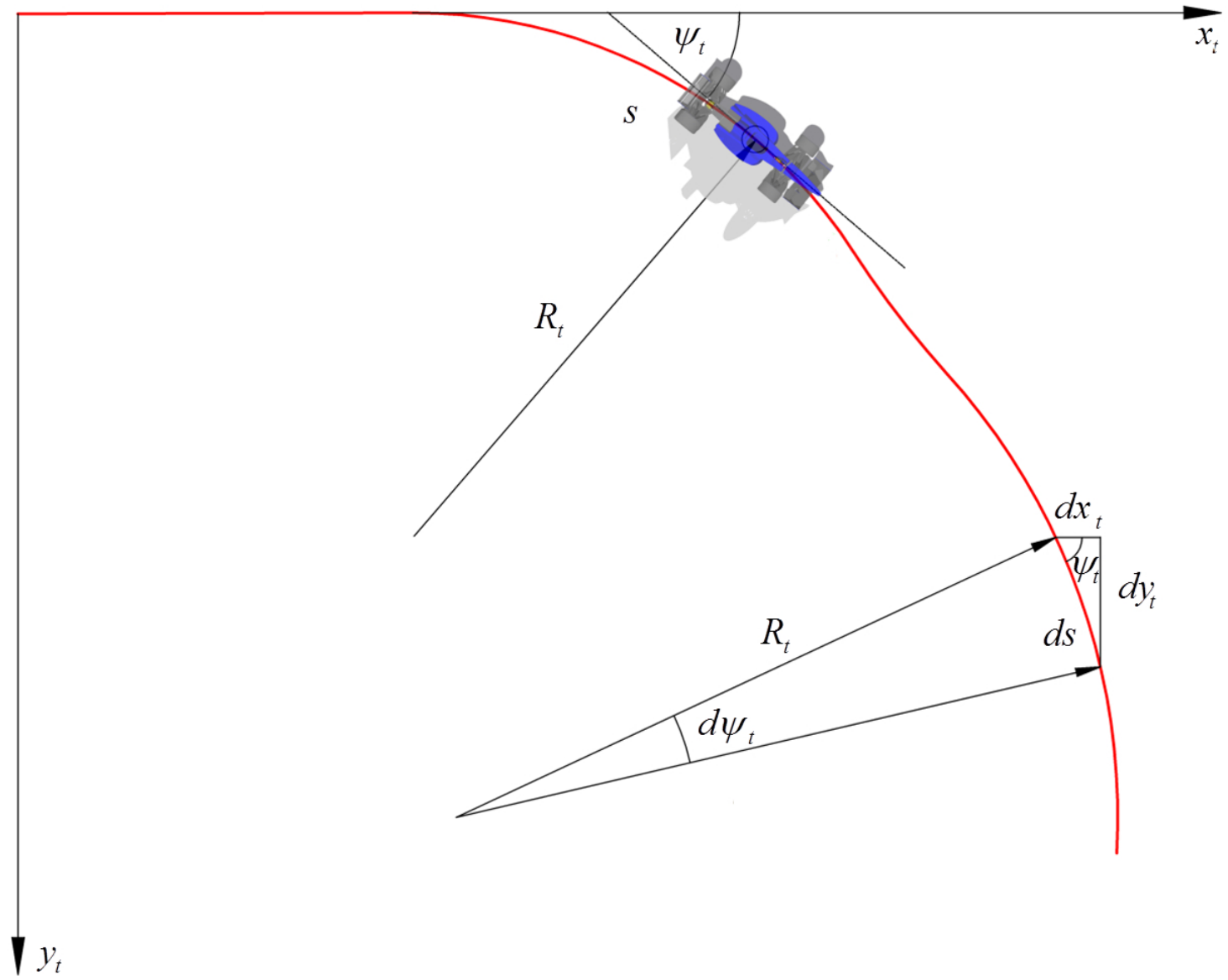

Figure 1. Plan view of the trajectory followed by the race car (adapted from [19]).

The reconstruction routine involves treating the vehicle as a particle and approximating its cornering radius from the measured lateral acceleration and forward speed data at each measured data point around the circuit. The calculated cornering radius allows the vehicle 
heading to be calculated, which can be combined with the vehicle speed data to determine the lateral and longitudinal coordinates of the vehicle's position relative to an initial starting origin.

At a number of points around the lap, with sample time $t$, the car's cornering radius is calculated by the standard equation of curvilinear motion, shown in Equation (1).

$$
R_{t}=v^{2} / A
$$

where $A$ is normal acceleration $\left(\mathrm{m} \cdot \mathrm{s}^{-2}\right)$, measured as vehicle lateral acceleration, $v$ is tangential velocity $\left(\mathrm{m} . \mathrm{s}^{-1}\right)$, measured as vehicle forward speed and $R_{t}$ is cornering radius (m).

If the plan view of the vehicle trajectory adapted from [19] is considered (Figure 1), three differential equations can be formed which govern the coordinates and heading of the car. Firstly, if the path distance covered by the car is denoted $s$ then:

$$
d s=R_{t} d \psi_{t}
$$

where $\psi_{t}$ is the heading angle of the car (rad.). Equation (2) can be rearranged to:

$$
d \psi_{t}=\frac{1}{R_{t}} d s=k_{t} d s
$$

where $k_{t}$ is the inverse of the radius $R_{t}$, termed the curvature $\left(\mathrm{m}^{-1}\right)$ equal to $A / v^{2}$ from Equation (1).

Again, with reference to Figure 1, the increment of the vehicle longitudinal and lateral coordinates can be expressed in the form of Equations (4) and (5).

$$
d x_{t}=\cos \left(\psi_{t}\right) d s
$$


$d y_{t}=\sin \left(\psi_{t}\right) d s$

A substitution for $d s$ is made using Equation (6) in Equations (3) to (5) to express them in terms of time, allowing the formation of three integrals, presented as Equations (7) to (9).

$$
\begin{aligned}
& d s=v d t \\
& \psi_{t}(t)=\int_{0}^{t} k_{t}(t) v(t) d t \\
& x_{t}(t)=\int_{0}^{t} \cos \left[\psi_{t}(t)\right] v(t) d t \\
& y_{t}(t)=\int_{0}^{t} \sin \left[\psi_{t}(t)\right] v(t) d t
\end{aligned}
$$

Where $x_{t}$ and $y_{t}(\mathrm{~m})$ are the longitudinal and lateral coordinates respectively.

Equation (6) is also integrated, giving vehicle path distance as a function of elapsed time:

$$
s(t)=\int_{0}^{t} v(t) d t
$$

Computation of these equations at discretised intervals around a lap of data provides an array of lateral and longitudinal coordinates that form the trajectory of the vehicle. However, a number of sources cause an error in the trajectory reconstruction:

1. The neglect of elevation change in the reconstruction;

2. Accelerometer offsets, resulting from incorrect setting or mounting of the accelerometer; 
3. A time proportional integration drift error;

4. Scaling errors;

5. Errors arising from vehicle slip.

The error arising in the trajectory computation is compensated by the "stretching" coordinate correction proposed by [19]. The error for heading and coordinates are evaluated from a first pass computation of Equations (7) to (10) over the total time $T$ with the error being equal to the difference compared to $2 \pi$ for the heading and to zero for both coordinates. An example of a trajectory computed from this first pass is illustrated as the top frame of Figure 2. These errors are then corrected on a per distance basis, by the following equations, which will yield the typical result illustrated in the lower frame of Figure 2:

$\bar{\psi}_{t}(t)=\int_{0}^{t}\left[k_{t}(t)-\frac{E_{\psi}}{S}\right] v(t) d t$
$\bar{x}_{t}(t)=\int_{0}^{t}\left\{\cos \left[\bar{\psi}_{t}(t)\right]-\frac{E_{x}}{S}\right\} v(t) d t$

$\bar{y}_{t}(t)=\int_{0}^{t}\left\{\sin \left[\bar{\psi}_{t}(t)\right]-\frac{E_{y}}{S}\right\} v(t) d t$

where, $\bar{\psi}_{t}, \bar{x}_{t}$ and $\bar{y}_{t}$ are the corrected heading angle (rad.), longitudinal and lateral coordinates $(m)$ and $S$ is the total lap length $(m)$, i.e. $S$ integrated over the whole lap. 

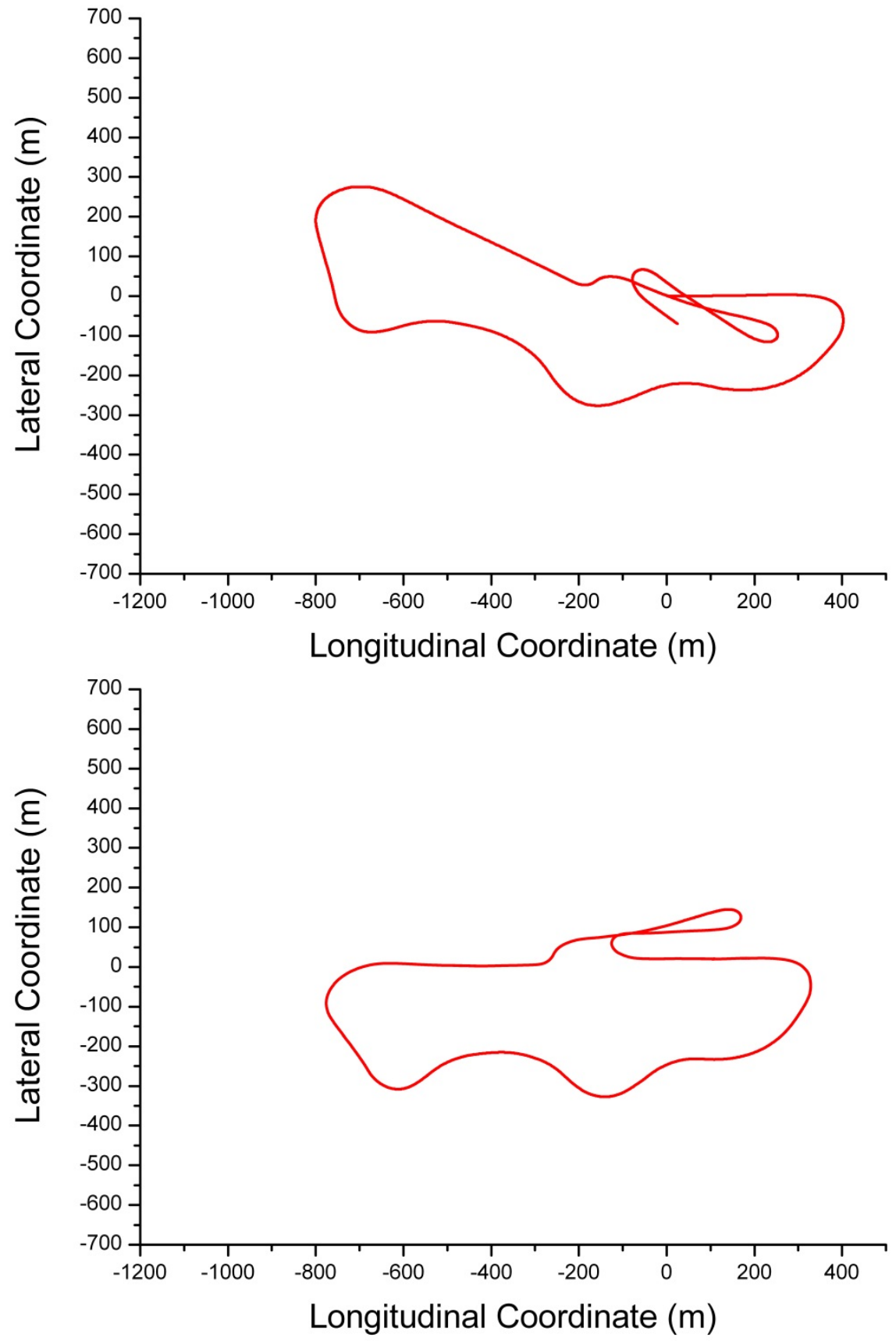

Figure 2. Inertial trajectory before correction (top) and with heading angle and coordinate stretching correction applied (bottom). 
These trajectory coordinates drive the vehicle model positioning during animated playback of the recorded data. At this stage of development, the attachment of the vehicle to a modelled ground plane, whilst tracking the approximated trajectory was simplified to attachment at a statically measured coordinate on the tyre (provided alongside the suspension joint location measurements by [18]). The vehicle body was also positioned with a fixed ride height and attitude. The various mechanisms affecting the location of the tyre contact patch and the vehicle body attitude and position, such as tyre deformation, steering and suspension travel and chassis compliance were not modelled or approximated for this first version of the application.

\subsection{Results}

To highlight the advantages of a visualisation tool for motorsport data analysis, a comparison is made between data presented in a typical format used within race teams and the results obtained from the visualisation tool. One example section of data is illustrated in Figure 3 , which shows vehicle speed vs cumulative distance for two laps at the same corner sequence (the "Esses"). The comparison illustrates the common occurrence of a driver braking later (the dashed lap) into the corner and ultimately losing time through its duration because of the car running wide at the corner apex, hindering the throttle application at the corner exit. This event is common and arises from a natural tendency of a driver to reduce the distance between their brake application and the entry of a given corner in an attempt to gain a time advantage through the corner as a whole. However, beyond a certain point, the effect is to increase the overall time through the corner and hinder performance through the subsequent section of track. It is evident that interpreting the data presented in this form is not intuitive and requires experience 
to ensure correct analysis. However, the visualisation tool reduces the abstraction between the data and the vehicle itself and allows the key aspects of the data to be understood more readily and quickly.

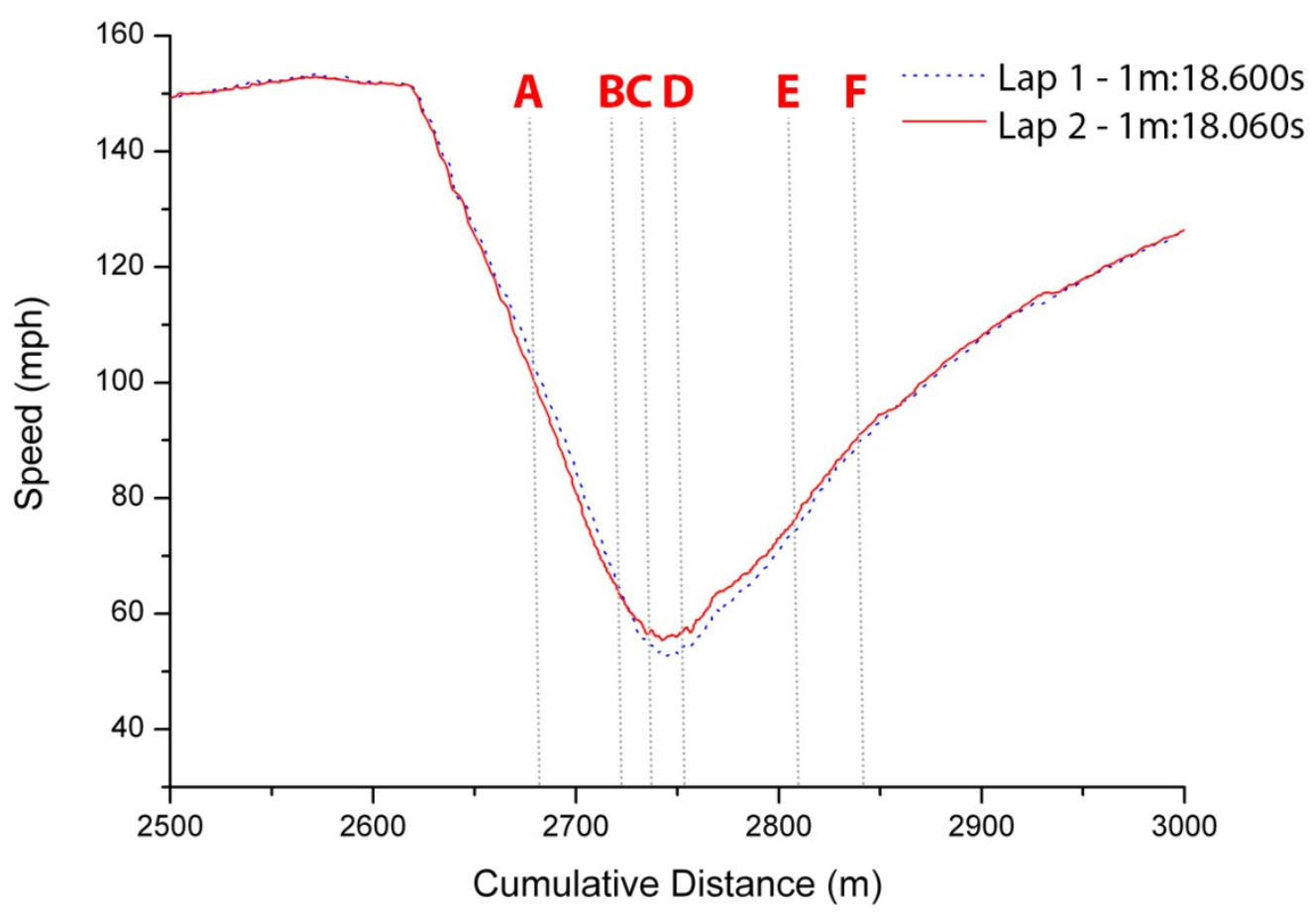

Figure 3. Graph of Speed (mph) vs. Cumulative Distance $(\mathrm{m})$ focussing on the "Esses" at the Donington Park Grand Prix circuit.

The series of screenshots in Figure 4 illustrate a number of key frames in the negotiation of the "Esses". A consideration of the screenshots should give the reader some impression of the assistance in visualising recorded data that the application can provide. Between frame $A$ and B, also marked on Figure 3, the blue car is seen to move ahead of the red, by later brake application into the corner sequence. At C and D, the wider line the blue car then follows is evident as a result of its later braking. Finally, E and $\mathrm{F}$ illustrate the red car gaining back and 
then increasing the advantage which it held prior to the corner sequence. The gain at $E$ and $F$ is made by virtue of the fact that the driving approach has allowed an earlier and more positive throttle application, resulting in faster acceleration from the corner minimum speed. 


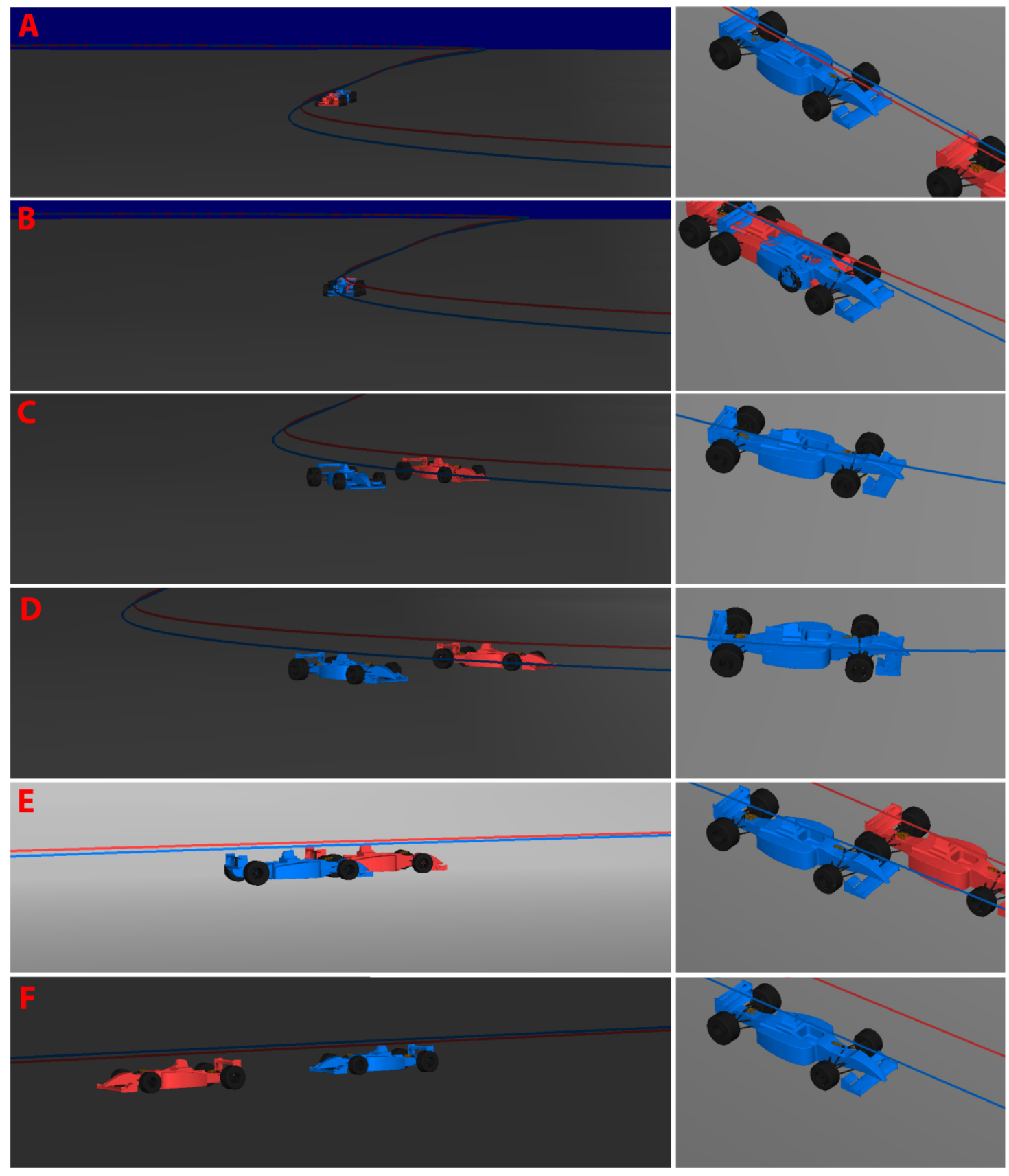

Figure 4. Sequence of frames showing the difference in approaches to the "Esses" over two laps. 
Comparison of the traditional approach to formatting motorsport data and the presentation of data within the visualisation tool highlighted a number of advantages for the latter approach including:

1. The abstraction between the car and data was removed;

2. The multi-dimensional nature of the data (e.g. vehicle velocity as a function of position in two dimensions) was illustrated, which it was not the case when solely using line graphs;

3. A visual focal point for discussion was presented in a form understandable by all levels of user.

Whilst this version of the application has illustrated its use for understanding some of the differences between varying driving approaches, there was still clear potential for improvement by demonstrating the motion of the vehicle and its suspension, one of the key areas of car performance analysed and adjusted by a race team for performance improvement. The placement of the actual vehicle model within its 3D environment also presented opportunity for development. The following section discusses the implementation of a kinematic model for recreation of the suspension motion, combined with a novel sensor to determine vehicle displacement.

\section{Development of the visualisation application}

The previous section has demonstrated that the visualisation tool concept could aid the user by reducing the abstraction between the recorded data and the race car and in doing so offered potential for assisting and accelerating the analysis of data, such that it could be more fully utilised at the race circuit. However, there was potential for increasing the benefits of the system by: 
1. Improving the accuracy of the trajectory calculation, such that the comparison of driver racing lines was more accurate;

2. Incorporating suspension motion to utilise the tool to visualise a number of additional areas of vehicle performance.

Suspension movement and steering input are routinely measured and recorded by race teams across all levels of motorsport typically using potentiometers or linear variable differential transformers (LVDTs) and was therefore a sensible representative basis on which to build the reconstructed vehicle motion visualisation tool described previously. The suspension system geometry of a vehicle is defined by the system joint locations. Examples of these joint locations are illustrated in Figure 5 by the reference abbreviations as referred to in [7], which are indicative of the joint name and its location on the car, e.g. FLUWBIR denoting Front Left Upper Wishbone Inboard Rear joint. If the vehicle body is considered static, several of these points, namely the inboard mounting points, are known relative to some origin on the chassis (and are therefore identified by uppercase abbreviations). 


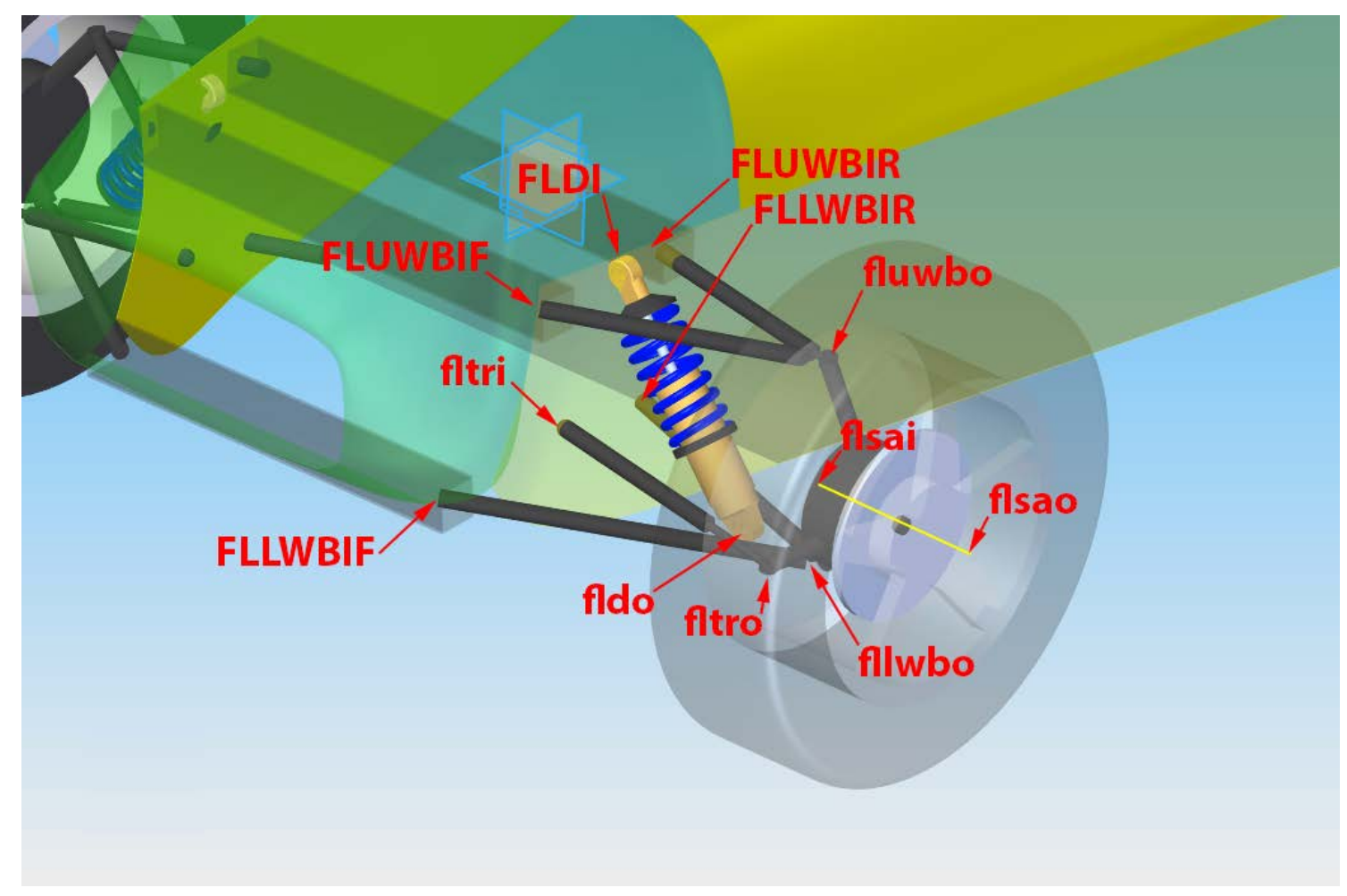

Figure 5. Screenshots from the Solid $E d g e \AA C A D$ package, illustrating the location of the coordinates involved in the calculation of the front left suspension kinematics.

The position of the vehicle's suspension components during the animation could then be determined using a triangulation approach based upon work described by $[22,23]$. This simple approach enabled a direct solution to be determined without the need for iteration, whilst maintaining scope for expansion by the introduction of suspension compliance [24].

Blundell and Harty [22] and Burgess et al. [23] describe the kinematic calculation approach in detail but a brief overview is presented here for completeness. The process described is for calculating the position of a single unknown point on the suspension system, and is repeated in turn for every unknown point, in most cases using calculated points as known points in subsequent calculations. At each animation frame or acquisition point, three points of known 
coordinates with known distance relationships (lengths) to an unknown point were used to find the coordinates of that unknown point. All known lengths were constant throughout a single data file with the exception of the damper length, which represented the varying boundary condition of the system, and which was provided by the acquired data.

The method for calculating a single unknown point's three coordinates is now presented. The calculation of point in question is started by equating the vector length between the unknown point and each of the three known points selected in terms of the individual coordinates. With three known points being used, this results in three equations of the form of Equation (14).

$$
|F L|=\sqrt{(K P x-U P x)^{2}+(K P y-U P y)^{2}+(K P z-U P z)^{2}}
$$

Where $K P$ is the known point, e.g. FLDI from Figure 5, UP is the unknown point, e.g. fldo from Figure 5 and $x, y$ and $z$ refer to the coordinates of each point whilst $F L$ is the fixed length between the known and unknown point.

Values for the coordinates of each known point and lengths to the unknown point are then substituted into three expressions of the form of Equation (14) and when expanded and rearranged yield three expressions of the form of Equation (15), where $\alpha, \beta$ and $\varepsilon$ are coefficients of the $U P x, U P y$ and $U P z$ terms respectively and $C$ is the resulting constant term.

$$
U P x^{2}+U P y^{2}+U P z^{2}+\alpha U P x+\beta U P y+\varepsilon U P z+C=0
$$


By subtracting one of the three equations of the form of (15) from another in three different combinations, three simultaneous equations of the form of (16) result in which the squared terms have been eliminated.

$\lambda U P x+\mu U P y+\xi U P z+D=0$

Where $\lambda, \mu$ and $\xi$ are coefficients and $D$ is the constant term resulting from the combination process described above.

The three simultaneous equations of the form of Equation (16) are then combined to eliminate terms and obtain expressions for, in this example, the $x$ and $y$ coordinates, each in terms of $z$, as in the form of Equations (17) and (18).

$U P x=\frac{\sigma U P z+E}{\phi}$

Where $\phi$ is the resultant coefficient of $U P x, \sigma$ is the coefficient of $U P z$ and $E$ is the resulting constant term.

$U P y=\frac{\chi U P z+F}{\psi}$

Where $\psi$ is the resultant coefficient of $U P y, \chi$ is the coefficient of $U P z$ and $F$ is the resulting constant term. 
Equations (17) and (18) are substituted, in this case, in place of UPx and UPy into the original Equation (14) to produce a quadratic equation in terms of a single variable $U P z$. This equation can be solved by the standard quadratic formula to yield two solutions which are then substituted in turn into Equations (17) and (18) to calculate the two possible solutions of the remaining two coordinates. Inspection of the two possible sets of coordinates based on their vector distance from the previous position of the point is then used to determine the correct solution.

Once calculated, coordinates for each suspension component were then used to position and orientate their model representations in the visualisation application, as illustrated by the development screen shot in Figure 6. These calculations were performed at each acquisition point from the recorded data and the screen update controlled to the same rate at which the data was acquired to result in the recreation of real time motion. There was also the potential for slowed playback to reveal high speed phenomena recorded by the data acquisition system. 

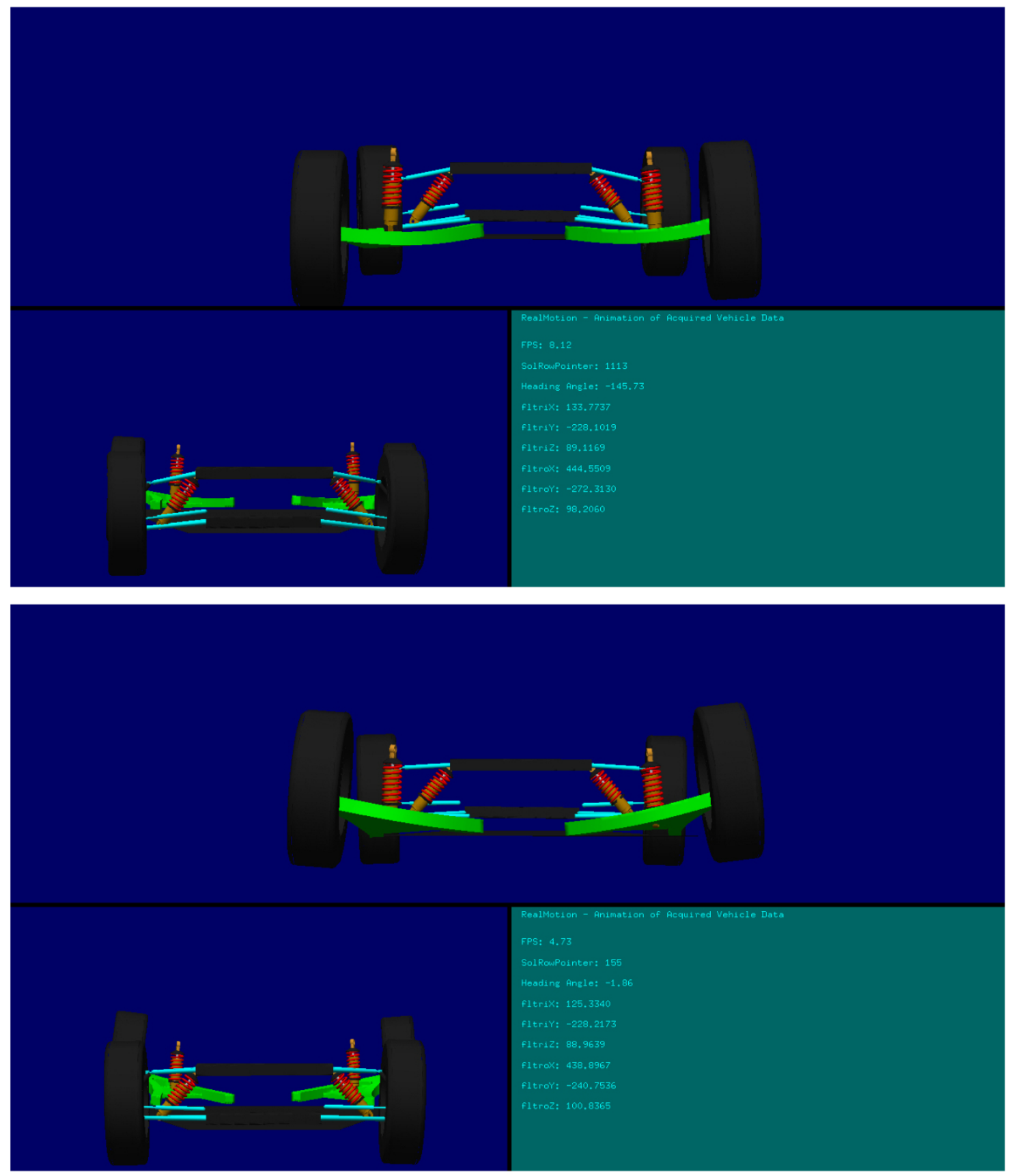

Figure 6. Screenshot from the suspension motion development application, illustrating the positioning of components to reflect the calculated solution of the suspension system (vehicle bodywork removed). 
The solution of the vehicle suspension points was conducted in a local coordinate frame, which subsequently required transforming into a world coordinate space, to give the impression of vehicle motion. The transformation in world coordinate space required the measurement of both vehicle trajectory and body attitude.

Vehicle trajectory reconstruction has previously been described using accelerometer and speed data, although it was shown to be insufficiently accurate for comparing vehicle trajectories. GPS based methods were found to be emerging as an alternative method of approximating trajectory, although a review of motorsport GPS technology suggested considerable shortcomings of the approach. GPS accuracy is typically worse than $3 \mathrm{~m}$ when used in its standard form [2] and although various approaches and technologies were found to be available to improve upon this performance, the best of these being the combination of inertial and GPS methods in a Kalman filter, which is still typically only able to achieve $1 \mathrm{~m}$ accuracy under the conditions imposed by the race circuit $[2,25]$. Professional opinion suggested that GPS was not employed in racing for accurate trajectory comparison because of the level of accuracy of its standard form and the practical limitations associated with its improvement [1, 21].

Consequently there was scope for the development of a new means of reconstructing the racing car's trajectory on circuit, the results of which could be visualised by the display routine developed previously.

A camera based system is described in the following section which was proposed for measurement of both vehicle trajectory and body motion/attitude. These data streams would subsequently be integrated into the visualisation application, to make the final development form tested in this work. 


\subsection{Vehicle positioning}

The preliminary approach to vehicle trajectory approximation and model positioning using inertial and speed data, described previously, was now replaced by a conceptual sensor system developed alongside this work which determined both vehicle position and attitude.

This sensor system was an optical, digital camera based sensor system, in which each sensor was referred to as an Optical Displacement Sensor (ODS). The system aimed to prove the fundamental concept of an approach which could theoretically offer improved accuracy over the practical application of GPS based methods and be manufactured at a lower cost and provide more flexibility than existing Correvit ${ }^{\circledR}$ type optical sensors [26]. It was also intended that the worth of the enhanced vehicle position and attitude data to the visualisation application be demonstrated.

The ODS system is described in more detail in [7], but to summarise, the system gave 2D (longitudinal and lateral) ground displacement and vertical ride height measurements at three locations by analysis of the captured images with autocorrelation and triangulation techniques respectively. A development ODS device is illustrated in Figure 7, consisting of a camera imaging the ground, surrounded by a ring LED illumination source, as well as a laser diode (obscured here by mounting plates), positioned at an angle and targeted to cast an image in the frame of the camera. 


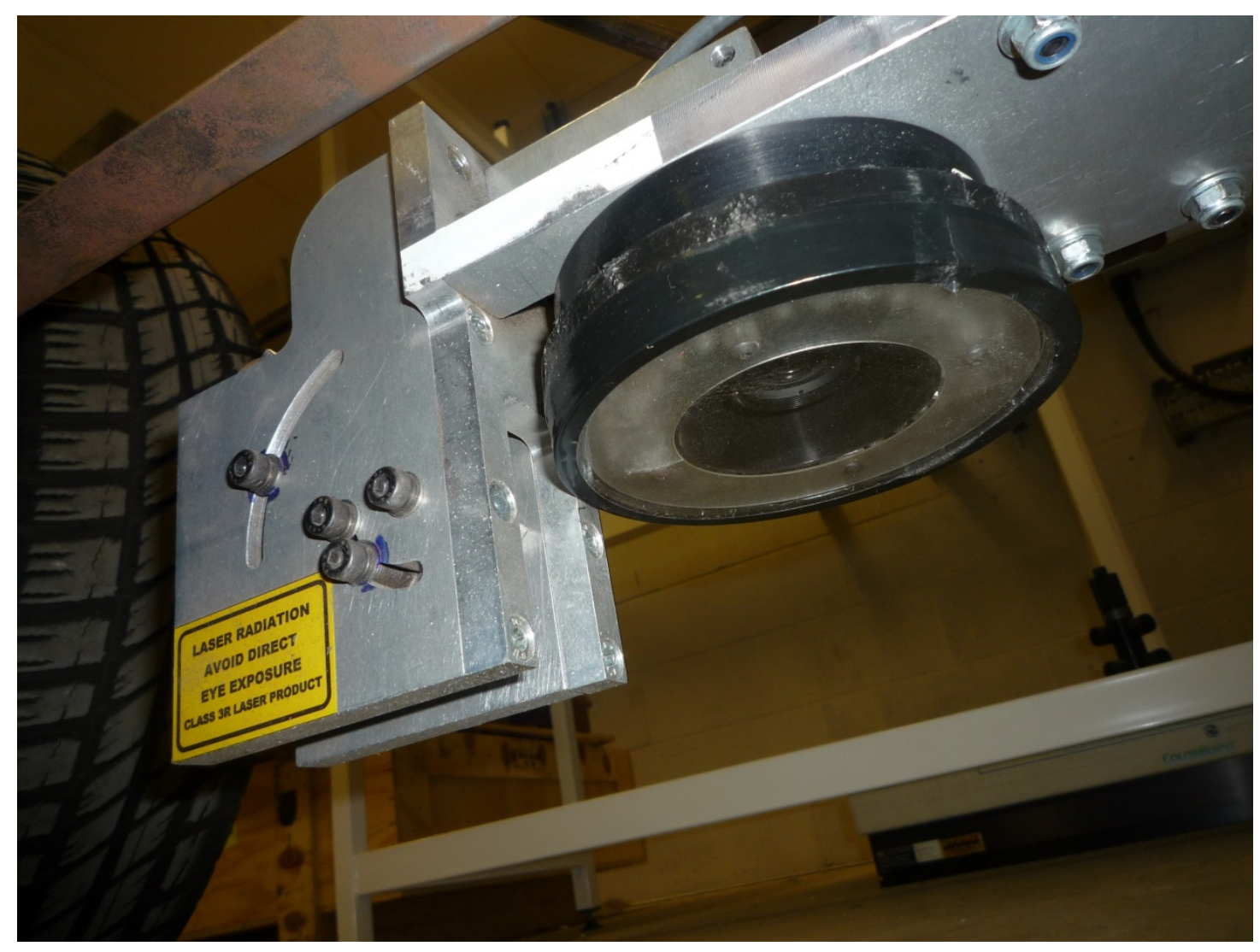

Figure 7. An optical displacement sensor (ODS) used as part of a system to determine vehicle trajectory and body motion.

The lateral and longitudinal displacements of the vehicle were determined by first producing a double pulse of LED illumination in each captured frame of the sensor. This illumination strategy resulted in an image containing repeated ground features captured during each pulse and separated by an offset resulting from the distance travelled by the car between the two pulses. The calculation routine involved shifting one copy of the image data relative to another and calculating a correlation between the two, such that the distance travelled by the sensor could be inferred by the number of pixels shifted to produce the strongest correlation. The ride 
height data from the sensor was measured by identifying the position of the imaged light spot of the angled laser diode, which moved across the frame with changes in ride height.

Three ODS devices were combined into a system, all located at known coordinate locations relative to the model geometry (one sensor at the front of the car and two at the rear). The displacement vector data from each of these devices were combined to determine an overall heading vector of the vehicle (and therefore yaw angle), whilst the ride height data was used to infer the vehicle pitch and roll angles. The difference between the measured ride height at the sensor locations and the value calculated by the kinematic method presented previously was used as an approximation of the vehicle chassis and tyre compliance.

The measurements of displacement and chassis ride height would allow for a plane approximation of the vehicle to be calculated and for this to drive the positioning of the vehicle model in animated playback, offering an enhanced impression of the vehicle's dynamic behaviour and an improved trajectory reconstruction for analysis and comparison. It was, however, envisaged that the ODS would ultimately need to be integrated with an absolute position sensor to reduce the impact of any integration errors of the approach, e.g. with a GPS receiver in a Kalman filter system. This prototype system would still illustrate additional information relating to the performance of the vehicle and potentially enhance the accuracy of the recreation over that driven by the accelerometer based trajectory reconstruction described previously. Selected initial results are presented in the following section. 


\subsection{Results}

The combination of ODS system vehicle trajectory and attitude approximation methods, with the display approach of the visualisation application described previously and the acquired data recorded from the Robin Hood kitcar test vehicle produced the final results of the work conducted to date. A number of tests were conducted with the kitcar to appraise both the ODS system and the development of the visualisation application. The tests included constant cornering radius, accuracy of distance measurement and repeatability tests over a simple circuit. Example results from the data gathered during the lap testing are presented here.

The following screenshots illustrate in more detail the visual impression of the vehicle motion afforded by the application by the inclusion of suspension calculation. The top frame of Figure 8 shows the car heading in a straight-line, with body level and suspension compression approximately equal each side. The lower frame of Figure 8 shows the car negotiating a right hand corner, which is clear from the animated steering angle. As one would expect, the car rolls to the left and the left side suspension compresses; this roll is clearly and rapidly visualised by the animation. 

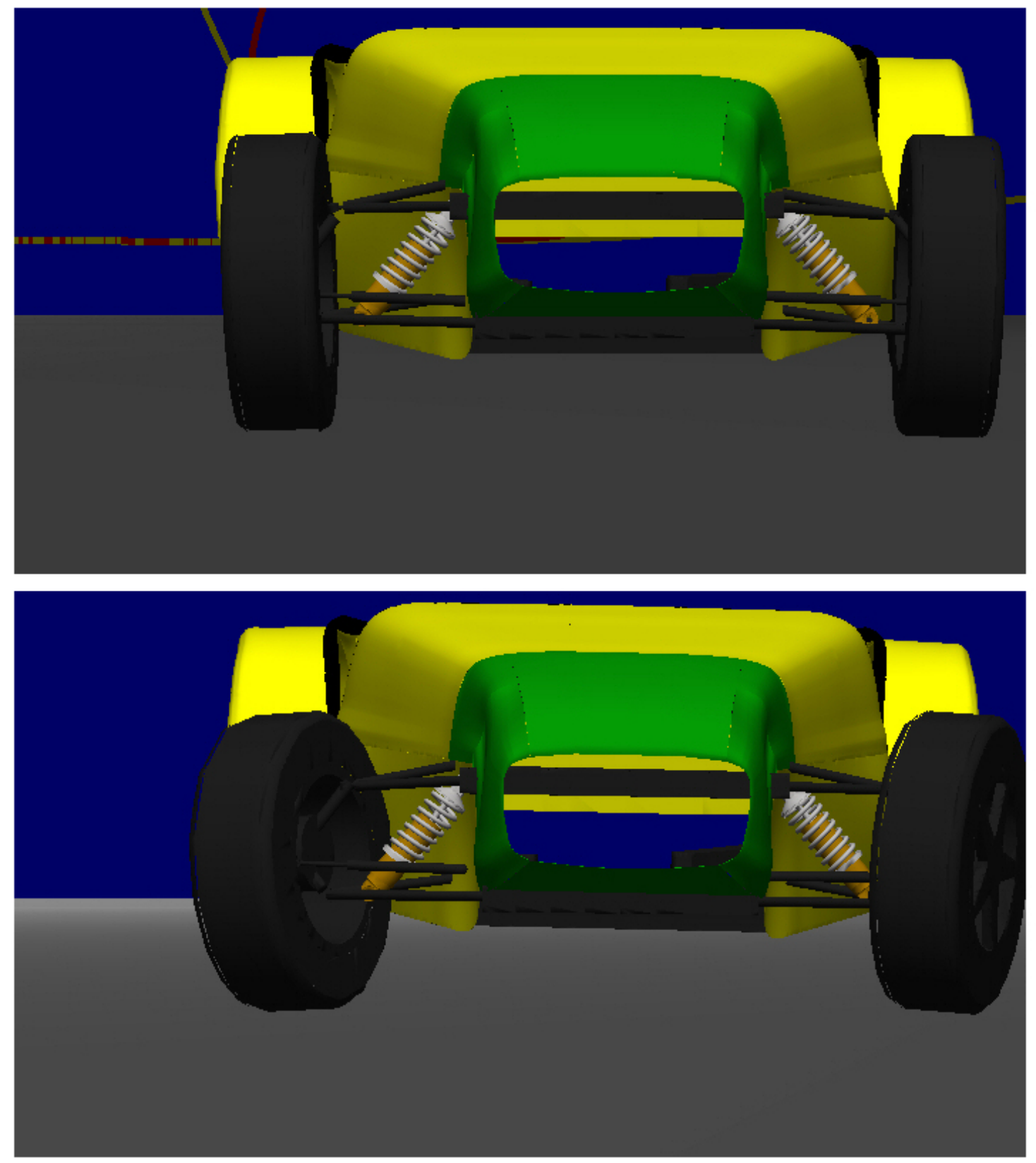

Figure 8. Screenshots illustrating the graphical visualisation of the steering and suspension motion, when suspension kinematics and ODS displacement data are included. In the top frame, the car is heading straight and in the lower frame it is cornering to its right. 
Figure 9 shows the most developed form of the visualisation application to date, with the ODS trajectory as well as suspension and body motion data integrated into a full animated display application, similar to that presented previously. The screen format depicted illustrates the system's ability to simultaneously present many different data to the user in a way which is not overwhelming and is intuitive to understand. The vehicle lap trajectory position and speed are illustrated in the bottom right of the screen, whilst the suspension data is shown in two screens, one giving a close scale impression of an area of interest whilst the other giving a farther scale impression, coupled with the vehicle's position on the circuit, allowing the differences between two laps of data to be visualised. It should also be noted, that when compared with Figure 4, it appears that the ODS trajectory reconstruction used here demonstrates more repeatable results than the inertial trajectory approximation routine used previously; this was also suggested in [7] with measured tests of both techniques recreating the same trajectory over several laps. 


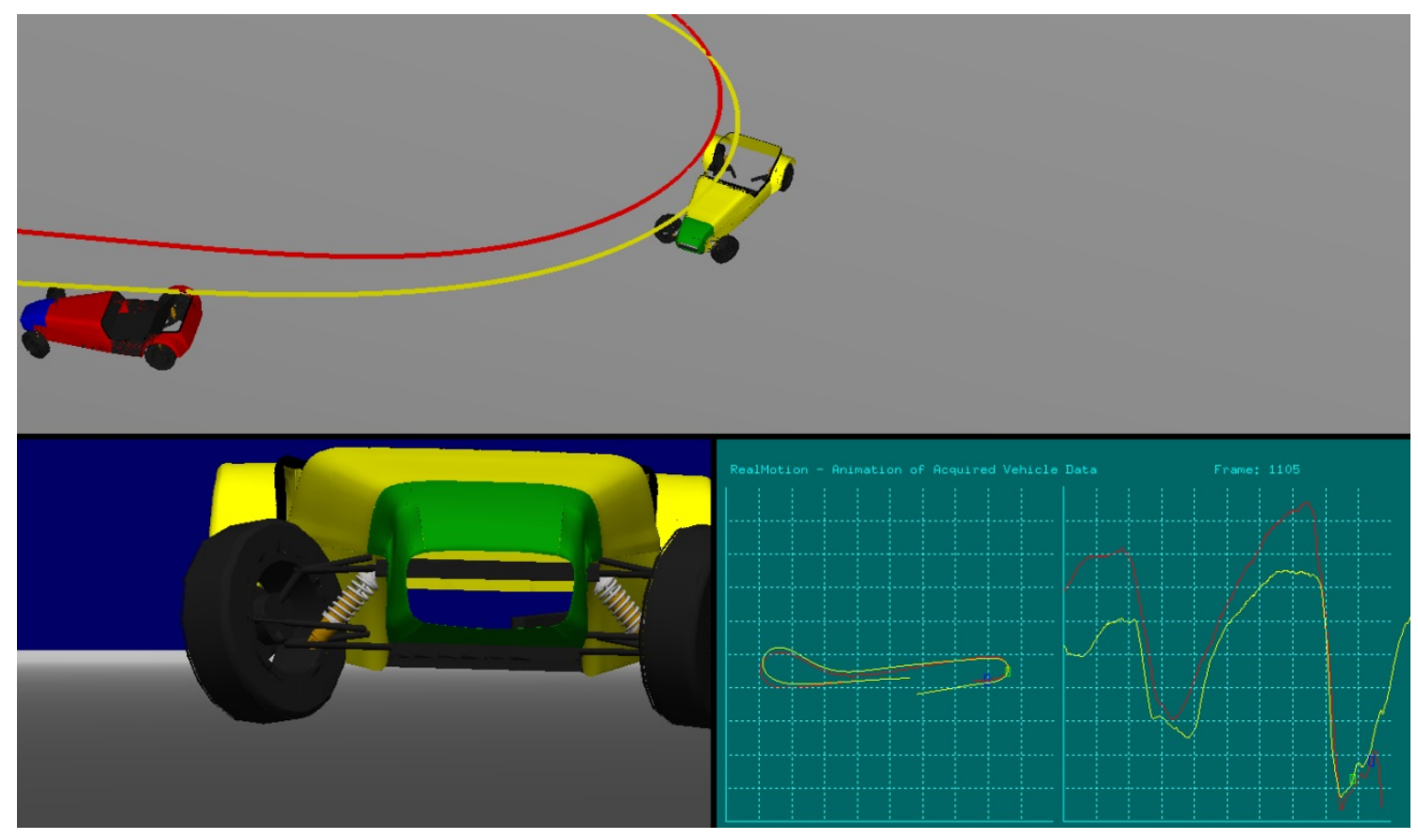

Figure 9. Screenshot depicting the most developed version of the visualisation application to date, incorporating suspension displacement and ODS trajectory data with graphed data.

\section{Conclusions}

The work presented in this paper represents a conceptual graphical visualisation system for acquired motorsport data, which employs graphics drawing approaches that allow data to be processed for animation at a rate quicker than real time. This rapid data processing speed makes the approach particularly viable and useable within in a motor racing environment. This conceptual system has demonstrated the proposed method as a beneficial alternative data display approach to the tools available in current commercial analysis software. The system achieves this without either the need for lengthy simulation or the complexity of introducing cameras and handling the image data they produce. It is also worth noting that the approach 
allows external viewpoints as well as views of vehicle components which may not be accessed or viewed by cameras.

Use of the new ODS system has also resulted in a significant improvement in trajectory reconstruction. The system has been demonstrated with a range of data, starting with that which is typical of the even the more junior levels of motor racing such as international and national championship support series and club racing. It has been shown to offer potential for allowing the user to better assimilate and understand the potentially extensive quantity of data collected and to do so more quickly and effectively than with graphs alone. Increasing the sophistication of the data provided to the system has allowed it to illustrate further insight into the performance of the vehicle in such a way that it does not overwhelm the user, as could very possibly be the case if the information were to be considered in its entirety employing only traditional graphing methods.

\section{References}

1. Templeman G. The Competition Car Data Logging Manual. Dorchester, UK: Veloce Publishing Ltd; 2008.

2. Segers J. Analysis Techniques for Racecar Data Acquisition. Warrendale, PA, USA: SAE International; 2008.

3. Purnell AJ. Innovative Computer Technology in Professional Motorsports. SAE. 1998; paper 983089.

4. Briggs G. Human Engineering. International Journal: Racecar Engineering, 2004, Vol. 14, No. 5, pp 38 - 42.

5. Vaduri S, Law EH. Development of an Expert System for the Analysis of Track Test Data. SAE. 2000; paper 2000-01-1628.

6. Martin BT, Law EH. Development of an Expert System for Race Car Driver \& Chassis Diagnostics. SAE. 2002; paper 2002-01-1574. 
7. Parker, M C. A system for aiding the user assimilation of acquired motorsport data. PhD Thesis. Loughborough University, Loughborough, UK; 2010.

8. Pi Research. Pi VIDS (Video Indexed Data System) User Guide. Cottenham, Cambridgeshire, UK: Pi Group Limited; 2000.

9. Moloney J, Coffey S, Perez A. Racing Applications for Digital Motion Analysis. SAE. 1998; paper 983086.

10. Teledyne. Vision, Flight Animation System. [Sales brochure] El Segundo, CA, USA: Teledyne Technologies; 2010.

11. SimAuthor. FlightViz ${ }^{\mathrm{TM}}$ Flight Visualisation Software: Debrief. [Corporate sales brochure] Boulder, CO, USA; 2010.

12. CEFA. Flight Animation Software, (http://www.cefaaviation.com/flight_data_animation.htm (Accessed February 2010).

13. Renault Sport Technologies. World Series by Renault: Formula Renault 3.52006 Technical Regulations. Les Ulis Courtaboeuf Cédex, France: Renault Sport Technologies; 2006.

14. Dallara. Dallara T05. [Technical drawings] Parma, Italy, Dallara Automobili; 2004.

15. Shreiner D. et al. OpenGL $₫$ Programming Guide: The Official Guide to Learning OpenGL ${ }^{\circledR}$, Version 2.1. 6th Ed. Boston, MA: Pearson Education Inc.; 2008.

16. Young MJ. Mastering ${ }^{\mathrm{TM}}$ Visual $\mathrm{C}++6$. Alameda, CA, USA: SYBEX, Inc.; 1998.

17. Martz P. OpenGL $®$ Distilled. Boston, MA, USA: Pearson Education, Inc.; 2006.

18. McBeath S. Competition Car Data Logging: A Practical Handbook. $1^{\text {st }}$ ed. Yeovil, Somerset, England: Haynes Publishing; 2002.

19. Casanova D, Sharp RS, Symonds P. Construction of Race Circuit Geometry from On-car Measurements. Proceeding of the Institution of Mechanical Engineers Part D. 2001, Vol 215.

20. Doogue M, Walsh M. The Design of a Track Map Based Data Acquisition System for the Dartmouth Formula Racing Team. Springfield, MA, USA: Analog Devices, Inc.; 1998.

21. McBeath, S. Competition Car Data Logging: A Practical Handbook . $2^{\text {nd }}$ Ed. Yeovil, Somerset, England: Haynes Publishing; 2008.

22. Blundell MV, Harty D. The Multibody Systems Approach to Vehicle Dynamics. Oxford, Oxfordshire, UK: Elsevier; 2004. 
23. Burgess MJ, et al. A Tool for Rapid Vehicle Suspension Design. SAE. 2004; paper 2004-01-3543.

24. Williams, S. Reducing Product Development Time Using Design Based Suspension Analysis. Conference paper. 3rd International Symposium on Multi-Body Dynamics: Monitoring and Simulation Techniques, Loughborough, England, 12th - 13th July 2004.

25. Oxford Technical Solutions. RT3000 Inertial and GPS Measurement System: Report from Silverstone F1 Test. [White paper] Upper Heyford, Oxfordshire, UK: Oxford Technical Solutions Ltd.; 2002.

26. Corrsys-Datron. The Physical Operating Principle of Correvit ${ }^{\circledR}$ Non-Contact Optical Sensors. [Information sheet] Wetzlar, Germany: Corrsys-Datron Sensorsysteme $\mathrm{GmbH} ; 2004$. 